\title{
High-speed photography of shock waves with an adaptive illumination
}

\author{
Vid Agrež, (i) Tomaž Požar, (1) and Rok Petkovšek* (1) \\ Faculty of Mechanical Engineering, University of Ljubljana, Aškerčeva 6, 1000 Ljubljana, Slovenia \\ ${ }^{*}$ Corresponding author: rok.petkovsek@fs.uni-lj.si
}

Received 24 January 2020; revised 15 February 2020; accepted 15 February 2020; posted 18 February 2020 (Doc. ID 388444); published 13 March 2020

\begin{abstract}
An adaptable, laser-diode-based illumination system was developed to simultaneously visualize the dynamics of slow and fast phenomena in optically transparent media. The system can be coupled with still or high-speed cameras and makes it possible to generate an arbitrary train of illumination pulses with a variable pulse duration, pulse energy, and an intrapulse delay with a temporal resolution of $12.5 \mathrm{~ns}$. Its capabilities are presented with selected illustrative visualizations of the dynamics of the shock waves and the cavitation entities generated after the laser-induced breakdown in water. () 2020 Optical Society of America
\end{abstract}

https://doi.org/10.1364/OL.388444

Provided under the terms of the OSA Open Access Publishing Agreement This Letter describes an adaptive illumination system to visualize at once a combination of slow and fast phenomena in transparent media. It is intended to be used as a highly adaptable light source for high-speed movie shadowgraphy, schlieren photography/cinematography [1-3], a background-oriented schlieren (BOS) technique [4], and high-speed photo-elasticity [5]. The slower phenomena are visualized frame by frame, similar to the conventional continuous illumination or single-pulse-per-frame illumination techniques, while faster phenomena are captured within each frame by using multiple probing pulses that "freeze" the rapid motion of the propagating object at different locations on the sensor. This illumination system can be used in connection with single-shot or high-speed video cameras.

In this context, slow phenomena are those where sharp images are obtained during the exposure time of a single frame, while changes are observed between the frames. The fast phenomena would be motion-blurred on the sensor during frame exposure and thus demand a short intraexposure probing pulse to freeze the object plane onto the sensor before the image of the object moves laterally more than 1 pixel.

An illustrative example that calls for such an illumination system is the qualitative visualization of phenomena following a laser-induced breakdown in liquids [6]. Such a breakdown is accompanied by an interesting interplay of cavitation structures and the emission of shock waves $[7,8]$. As for the speed at which optical changes are expected, the cavities can be considered as structures with a slow dynamic character, while shock waves can be assigned a fast character [9].
For example, assuming no optical magnification, a shock wave propagating in water acoustically at $1500 \mathrm{~m} / \mathrm{s}$ takes about $13 \mathrm{~ns}$ to traverse a single pixel $(20 \mu \mathrm{m} \times 20 \mu \mathrm{m})$ of a typical high-speed camera. To capture sharp images of shock wave dynamics with continuous illumination, this requires a camera with a 10 ns exposure, a high frame rate of more than $10 \mathrm{Mfps}$ (mega frames per second) with preferably a megapixel resolution or more, which is only available with very complex systems based on an image intensifier. Current commercially available digital high-speed video cameras reach a frame rate of up to $21 \mathrm{kfps}$ at a 1 megapixel resolution and a rolling memory buffer with a practically unlimited number of frames (Fastcam SA-Z, Photron, Japan) or up to $10 \mathrm{Mfps}$ at a 50 kilopixel resolution, an exposure time of $50 \mathrm{~ns}$, and a maximum of 256 frames (Hyper Vision HPV-X2, Shimadzu, Japan). Despite the ongoing progress [10], 10-ns-exposure high-speed cameras with megapixel resolution are still far from being commercially available.

An alternative approach is to modify the illumination part of the illumination-detection visualization system, while, if needed, preserving the benefits of fast-camera acquisition. This is a more affordable approach, because it is often sufficient to capture the object of interest, e.g., a shock wave [11] or a cavitation-bubble boundary [12], multiple times during a single exposure, where it is sufficient to use a simple still camera.

Different light sources [lasers or laser diodes and lightemitting diodes (LEDs)] and various pulse-generation strategies have been employed for this purpose. Multiple pulses can be generated by splitting a single pulse into multiple pulses and delaying them either with optical fibers of different lengths [13] or by using different free-space paths [11]. These techniques lack adaptability, due to mechanical realization of the intrapulse delays.

A drawback of using a typical laser source like Q-switched [13] or mode-locked lasers [11,14] as the light source is the inflexibility of the pulse modulation and the speckled, nonhomogeneous spatial profile. The illumination can be switched on and off relatively rapidly with LEDs $[3,12,15]$, but to achieve high-brightness pulses with durations shorter than $100 \mathrm{~ns}$, laser diodes are preferred, especially when unwanted light sources at other wavelengths have to be filtered out. 
The presented, laser-diode-based illumination system is highly adaptable since it allows the generation of camerasynchronized, arbitrary trains of pulses with a variable pulse duration, adjustable energies per pulse, and an inconstant intrapulse idle time with a temporal resolution of $12.5 \mathrm{~ns}$.

The presented high-speed photography setup is shown in Fig. 1. The adaptive illumination system is built from a laser diode and a custom high-speed electronics driver based on a computer-controlled field-programmable gate array (FPGA) module that produces pulses as short as $16 \mathrm{~ns}$ covering the whole bandwidth of repetition rates from single shot to approximately $30 \mathrm{MHz}$. The duration of the illumination pulses and the timing can be set from pulse to pulse through the computer interface. The control electronics can be synchronized with an arbitrary external trigger.

The light source is a commercially available single-emitter laser diode module coupled to a standard multimode $125 \mu \mathrm{m}$ fiber that emits light at a nominal wavelength of $793 \mathrm{~nm}$. This wavelength offers a very good trade-off between power, brightness, camera-sensor sensitivity, and the transmissivity of water [16]. Due to the fiber-based architecture, power scaling can be easily realized, which further ads to the flexibility of the system and its capability to adapt to different requirements for high-speed photography. The illumination system allows for synchronization with the excitation laser and the still camera for the multi-illumination, single-frame (MI:SF) approach or directly with the output sync signal from a high-speed camera for a multi-illumination, multiframe (MI:MF) approach.

Both types of approach were tested. For MI:MF, a high-speed camera (Fastcam SA-Z, Photron, Japan) was used that allowed a $640 \times 280$ resolution at $100 \mathrm{kfps}$. For MI:SF, an industrial still

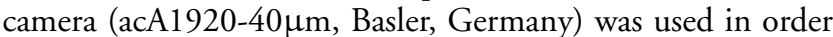
to show the benefits of multiple short-pulse illumination while capturing the images at higher resolution up to $1920 \times 1200$.

The illumination system and both cameras were applied to the optical setup for shadowgraphy, which is easily convertible to the schlieren setup for enhanced shock wave photography. The setup consists of three lenses $\left(\mathrm{L}_{1}-\mathrm{L}_{3}\right)$ and a camera objective $\mathrm{L}_{4}$. Lens $\mathrm{L}_{1}$ serves as the collimator, and $\mathrm{L}_{2}$ focuses the light on the spot for the spatial filter (in our case a knife edge), while $\mathrm{L}_{3}$ collimates the filtered light again and allows for easier camera placement.

To induce an optical breakdown, a Q-switched Nd:YAG laser was used to generate 6 ns pulses (FWHM) with an energy of $10 \mathrm{~mJ}$ that were focused into distilled water.

The performance of the presented illumination system is best demonstrated by illustrative visualizations of the shock waves and cavitation bubbles.

Figure 2(a) presents the growth of the laser-induced cavitation bubble and the emission of the shock wave in water. The shadowgram was acquired using the MI:SF approach and the optical layout shown in Fig. 1 upon the removal of the knifeedge-type aperture [3]. The shock waves are labeled with S\# and the boundaries of the cavitation bubbles with B\#, where \# stands for the number of the consecutive pulses. Four $16 \mathrm{~ns}$ pulses at a peak power of $8 \mathrm{~W}$, launched $1.25 \mu \mathrm{s}, 2.0 \mu \mathrm{s}, 17.0 \mu \mathrm{s}$, and $54.5 \mu$ s after the breakdown, outline the boundary between the gaseous bubble interior and the surrounding liquid. The first two pulses also capture the spherically propagating shock wave. Except at the very center, the bubble deflects the probing light and thus appears black. The integrated light level of all

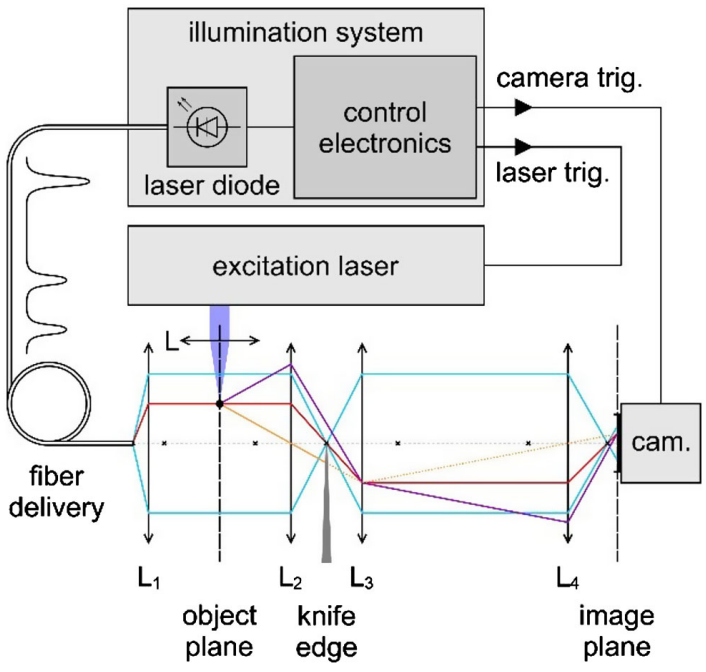

Fig. 1. Schematic of the setup for high-speed photography. Black dot, object deflecting the rays. Blue rays, outermost rays, defining the field of view. Red ray, the path of the nondeflected ray. Magenta ray, the path of the ray, deflected away from the knife edge. Orange ray, the path of the ray, deflected towards and obstructed by the knife edge. Labels, positive lenses $\left(\mathrm{L}_{i}\right)$.

four pulses is close to the saturation level of the camera, which means the bubble frozen by the first pulse appears black, while its increments between subsequent pulses are seen discretely brighter, giving a clear impression of the bubble's growth. A similar breakdown event was visualized in Fig. 2(b), except that this time seven pulses, generated $1.25 \mu \mathrm{s}, 2.0 \mu \mathrm{s}, 11.4 \mu \mathrm{s}$, $30.1 \mu$ s, $48.9 \mu \mathrm{s}, 67.6 \mu$ s, and $86.4 \mu$ s after the breakdown, with a smaller energy, were used for discretized illumination. Due to light accumulation, more pulses make the shock wave less pronounced. A large portion of the bubble radius-versus-time curve can be easily extracted from a single figure, which is important in the studies of laser-and acoustically nucleated cavitation bubbles [12]. Likewise, the shock wave velocity as a function of distance from the emission origin can also be determined based on a single image [17].



(b)

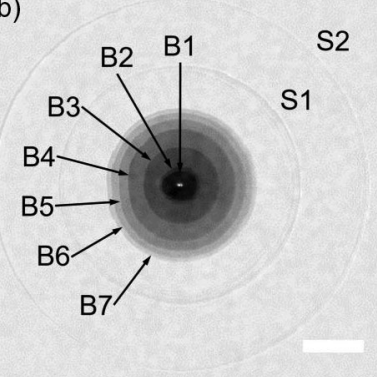

Fig. 2. Initial growth of a laser-induced cavitation bubble in water and shock wave emission illuminated with multiple visualization pulses using the MI:SF approach. (a) Four probe pulses at $1.25 \mu \mathrm{s}$, $2.0 \mu \mathrm{s}, 17.0 \mu \mathrm{s}$, and $54.5 \mu \mathrm{s}$ after laser breakdown. (b) Seven probe pulses at $1.25 \mu \mathrm{s}, 2.0 \mu \mathrm{s}, 11.4 \mu \mathrm{s}, 30.1 \mu \mathrm{s}, 48.9 \mu \mathrm{s}, 67.6 \mu \mathrm{s}$, and $86.4 \mu$ s after the laser breakdown. Labels, boundaries of cavitation bubbles $(\mathrm{B} \#)$ and shock waves $(\mathrm{S} \#)$, where \# stands for the number of the consecutive illumination pulse. White bar, $1 \mathrm{~mm}$. 
To remedy the problem of insufficient shock wave contrast with respect to the homogeneous background, the schlieren technique (knife inserted in Fig. 1) should be used. Allowing for adequate background illumination, the more the knife edge cuts the focus, the better the shock wave contrast. To demonstrate the schlieren technique to visualize shock waves, the shock wave was sent to be reflected from the plane targets of different acoustic impedances, such that the shock wave is reflected by changing the polarity [18] [Fig. 3(a), free-surface of water, subfigure rotated by $90^{\circ}$ ], without changing the phase [Fig. 3(b), aluminum], or with no reflection [Fig. 3(c), water impedance-matched transparent polymethylpentene (PMP)]. The transmission of the shock wave into the solid PMP, where it propagates faster, can also be observed in the right-most part of Fig. 3(c), demonstrating the ability of the visualization system to also capture shock waves in transparent solids [19]. Reflection from the aluminum surface, visualized in Fig. 3(b), belongs to the "rigid bottom case," where the shear velocity of the solid is larger than the water velocity. Here, the expected two head waves connecting the P-wavefront in the liquid with the $\mathrm{S}$ - and P-wavefronts in the solid [20] were also successfully visualized.

Moreover, solely based on the shock wave brightness and prior knowledge of the direction of the shock wave propagation, we can distinguish between the compressional or rarefactional phases of shock waves. See, for instance, the pixel-level of the shock waves marked with A and C in Fig. 3(a). The opposite polarity originates from the opposite slopes of the trailing parts of the initial and reflected shock waves traveling in the same direction. Such studies are applicable in the area of shock wave (acoustic wave) interactions with the interfaces [21-24].

Focusing of the shock waves [24-26] is exploited in medicine, either as a therapeutic technique employing low-intensity focused ultrasound (LIFU) [27] or high-intensity focused ultrasound (HIFU) [28], where tissue in the focal volume is either stimulated (LIFU) or destroyed by heating (HIFU). Increasing the pressure amplitude brings us to destructive ultrasonic histotripsy [29], where the targeted tissue experiences mechanical breakdown due to intense sonification. An example of shock wave focusing is captured in Fig. 4(a) using the MI:SF approach. The shock wave was generated by an optical breakdown. A portion of this transient was reflected from a solid acoustic mirror (thick black curve) and refocused on the axis between the apex of the spherical mirror and the optical focus $(\mathrm{Fo})$. Four

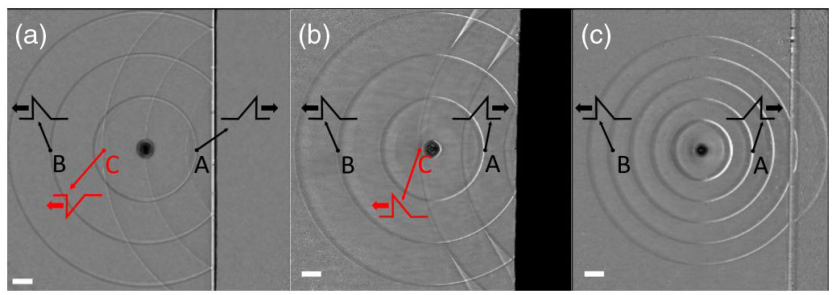

Fig. 3. Reflection of water-born shock wave from media with various acoustic impedances. (a) Free surface of water in vertical orientation (phase reversal at reflection), (b) aluminum plate (no phase reversal), and (c) PMP plate (no reflection). (a)-(c) Schlieren photographs in the MI:SF approach with the vertical knife filtering were illuminated with (a), (b) a triple and (c) a quintuple train of probe pulses. The part of the shock wave propagating towards the boundary is marked with A (black), the one traveling away from the boundary with $B$ (black), and the reflected one with $C$ (red). The shock waves were generated in water during optical breakdown. White bar, $1 \mathrm{~mm}$.
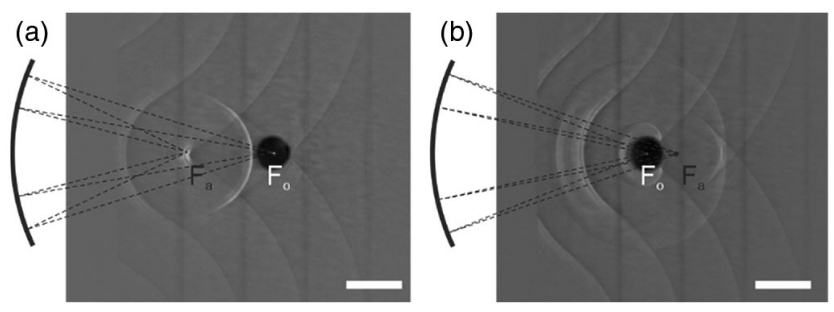

Fig. 4. Shock wave refocusing in water at the position (Fa): (a) between the initial breakdown and the concave acoustic mirror and (b) farther away from the optical focus (Fo) imaged using the MI:SF approach. (a) Visual determination of the acoustic focus. (b) Shock wave scattering on a growing cavity. The thick solid curve marks the position of the acoustic mirror and the thin dashed lines show the path of four selected rays from the shock origin to the acoustic focus. White bar, $1 \mathrm{~mm}$.

probe pulses with constant intrapulse delay illuminated the converging shock wave, clearly locating the acoustic focus $(\mathrm{Fa})$. An interesting phenomenon was captured, i.e., the Gouy phase shift of $\pi$, characteristic for spherical focusing of the pressure waves after they had already overpassed the focus $[30,31]$.

The presented high-speed photography of shock waves using an adaptive illumination in the MI:SF approach is also capable of revealing other wave-related phenomena, such as wave diffraction/scattering on acoustic obstacles. Figure 4(b) was taken using the same illumination settings as in Fig. 4(a). The generation of the shock wave was also similar, except that the optical breakdown was created closer to the apex of the acoustic mirror, moving the acoustic focus to the right. Figure 4(b) presents the interaction of the converging shock wave with a growing cavitation bubble. The reflection from the bubble gives rise to a new, nearly spherical transient. On the axis, the transmitted shock wave experiences a short time-delay due to the spherical obstacle. The creeping waves propagating on the surface of the cavity can also be visualized [32].

So far, we provided examples obtained with the MI:SF approach, i.e., single shadowgraphs/schlieren images illuminated with multiple pulses. Further versatility of the illumination system will be demonstrated with a MI:MF video approach, where slow phenomena are followed frame by frame, while fast phenomena are frozen multiple times within each frame. The experimental configuration to obtain Fig. 5 is the same as in Fig. 4. Figure 5 presents six selected frames from the high-speed schlieren cinematography with a frame rate of $100 \mathrm{kfps}$ using a train of six illumination pulses per frame with an intraframe separation of $250 \mathrm{~ns}$. Watching the video (Visualization 1) gives information about the evolution of cavitation structures, while inspecting each frame separately clearly illustrates the propagation of shock waves. Frame 1 [Fig. 5(a)] shows the inception of the main cavitation bubble and the release of a single breakdown-generated shock wave pulse-frozen six times. At $20 \mu \mathrm{s}$ after the breakdown [frame 2, Fig. 5(b)], the shock wave had been reflected from the acoustic mirror and overpassed the acoustic focus. Small cavities were generated by the shock wave in the preheated cone of the incoming laser pulse. The main bubble reaches its maximum size in frame 16 [Fig. 5(c)] and collapses about $250 \mathrm{~ns}$ before the first illumination pulse gives its stamp (innermost circle) in frame 30 [Fig. 5(d)]. At collapse, another shock wave is launched, which again generates small bubbles in the incoming laser cone, 




Fig. 5. (a)-(f) Selected frames from the high-speed schlieren video providing a visual insight into the bubble and shock wave dynamics after an optical breakdown in water near a concave acoustic mirror (see Visualization 1). The video was obtained with a frame rate of $100 \mathrm{kfps}$ using the MI:MF approach. White bar, $1 \mathrm{~mm}$.

after its reflection from the acoustic mirror. The location of the acoustic focus can be straightforwardly determined in frame 31 [Fig. 5(e)]. The last image picked [frame 39, Fig. 5(f)] displays, in addition to another shock wave emitted during the second collapse of the main bubble, a dislocated shock wave generated at the position of the acoustic focus due to the collapse of the acoustically generated cavitation cloud.

In conclusion, the demonstrated high-speed multiillumination of a single photograph or multiple video frames offers a unique way of studying shock waves and their interplay with cavitation structures in transparent media or other highspeed phenomena that affect its density. The full power of the laser-diode-based illumination system is its high adaptability, which allows the generation of camera-synchronized, arbitrary trains of probe pulses with a variable pulse duration, pulse energy, and an intrapulse delay with a temporal resolution of $12.5 \mathrm{~ns}$. Due to the high repeatability of the studied processes, we can use the presented approach as an online visualization tool to determine the position of both the optical and acoustic focus. In addition to the already mentioned applications, we foresee that the presented visualization technique will be well received also in the studies of laser shock processing [33], cavitation erosion [34], and cell transfection [35].

Funding. Javna Agencija za Raziskovalno Dejavnost RS (L2-8183, L2-9240, L2-9254, P2-0270); European Regional Development Fund.

Acknowledgment. This Letter is in part the result of work in the implementation of the SPS Operation entitled Building Blocks, Tools and Systems for Future Factories_-GOSTOP.

\section{REFERENCES}

1. P. K. Panigrahi and K. Muralidhar, in Schlieren and Shadowgraph Methods in Heat and Mass Transfer (Springer, 2012), pp. 23-46.

2. G. S. Settles, Schlieren and Shadowgraph Techniques: Visualizing Phenomena in Transparent Media (Springer, 2001).

3. G. S. Settles and M. J. Hargather, Meas. Sci. Technol. 28, 042001 (2017).

4. S. Yamamoto, Y. Tagawa, and M. Kameda, Exp. Fluids 56, 93 (2015).

5. K. Ramesh, Digital Photoelasticity: Advanced Techniques and Applications (Springer, 2012).

6. A. Vogel, I. Apitz, S. Freidank, and R. Dijkink, Opt. Lett. 31, 1812 (2006).

7. O. Supponen, D. Obreschkow, P. Kobel, M. Tinguely, N. Dorsaz, and M. Farhat, Phys. Rev. Fluids 2, 093601 (2017).

8. R. Petkovšek and P. Gregorčič, J. Appl. Phys. 102, 044909 (2007).

9. K. Johansen, J. H. Song, K. Johnston, and P. Prentice, Ultrasonics 73, 144 (2017).

10. R. Kuroda and S. Sugawa, Proc. SPIE 10328, 1032802 (2017).

11. P. Gregorčič and J. Možina, Opt. Lett. 36, 2782 (2011).

12. C. T. Wilson, T. L. Hall, E. Johnsen, L. Mancia, M. Rodriguez, J. E. Lundt, T. Colonius, D. L. Henann, C. Franck, Z. Xu, and J. R. Sukovich, Phys. Rev. E 99, 043103 (2019).

13. T. Perhavec and J. Diaci, Strojniski Vestnik 56, 477 (2010).

14. A. Vogel, S. Busch, and U. Parlitz, J. Acoust. Soc. Am. 100, 148 (1996).

15. C. E. Willert, D. M. Mitchell, and J. Soria, Exp. Fluids 53, 413 (2012).

16. S. Kedenburg, M. Vieweg, T. Gissibl, and H. Giessen, Opt. Mater Express 2, 1588 (2012).

17. R. Petkovšek, J. Možina, and G. Močnik, Opt. Express 13, 4107 (2005).

18. H. Hosseini, S. Moosavi-Nejad, H. Akiyama, and V. Menezes, Appl. Phys. Lett. 104, 103701 (2014).

19. P. E. Bloomfield, W. J. Lo, and P. A. Lewin, IEEE Trans. Ultrason. Ferroelectr. Freq. Control 47, 1397 (2000).

20. J. M. Carcione and H. B. Helle, Geophysics 69, 825 (2004).

21. K. Ohtani and T. Ogawa, Mech. Eng. J. 3, 16 (2016).

22. L. P. Geldart and R. E. Sheriff, Problems in Exploration Seismology and Their Solutions (Society of Exploration Geophysicists, 2004).

23. D. K. L. Don-Liyanage and D. C. Emmony, Appl. Phys. Lett. 79, 3356 (2001).

24. V. Eliasson, M. Mello, A. J. Rosakis, and P. E. Dimotakis, Shock Waves 20, 395 (2010).

25. G. N. Sankin, Y. F. Zhou, and P. Zhong, J. Acoust. Soc. Am. 123, 4071 (2008).

26. N. Apazidis and V. Eliasson, Shock Focusing Phenomena: High Energy Density Phenomena and Dynamics of Converging Shocks (Springer, 2018).

27. C. Y. Tao, G. Guo, Q. Y. Ma, J. Tu, D. Zhang, and J. M. Hu, J. Appl. Phys. 122, 014901 (2017)

28. J. E. Kennedy, G. R. ter Haar, and D. Cranston, Br. J. Radiol. 76, 590 (2003).

29. V. A. Khokhlova, J. B. Fowlkes, W. W. Roberts, G. R. Schade, Z. Xu, T. D. Khokhlova, T. L. Hall, A. D. Maxwell, Y. N. Wang, and C. A. Cain, Int. J. Hyperthermia 31, 145 (2015).

30. A. A. Kolomenskii, S. N. Jerebtsov, and H. A. Schuessler, Opt. Lett. 30, 2019 (2005).

31. T. Požar, M. Halilovič, D. Horvat, and R. Petkovšek, Appl. Phys. A 124, 112 (2018).

32. C. X. Pei, T. Fukuchi, H. T. Zhu, K. Koyama, K. Demachi, and M. Uesaka, IEEE Trans. Ultrason. Ferroelectr. Freq. Control 59, 2702 (2012).

33. K. Ding and L. Ye, Laser Shock Peening-Performance and Process Simulation (CRC Press, 2006).

34. A. Philipp and W. Lauterborn, J. Fluid Mech. 361, 75 (1998).

35. T. Kodama, M. R. Hamblin, and A. G. Doukas, Biophys. J. 79, 1821 (2000).

Disclosures. The authors declare no conflicts of interest. 